\title{
Experimental Evaluation of the Plastic Zone at Crack Tip by Caustics
}

\author{
B.G. Badalouka* and G.A. Papadopoulos* \\ Department of Engineering Science, Section of Mechanics, National Technical University of Athens, GR-157 73, \\ Zografou Campus, Athens, Greece
}

\begin{abstract}
The study of this paper was to determine the plastic zone dimension at the crack tip by the experimental method of caustics. The caustics shape at the crack tip depends on plastic zone which is small for brittle materials and large for ductile ones. The plastic zone dimensions depends on the crack length, the plates thickness and the loading level. The experimental method of caustics is an excellent tool for the plasticity and stress intensity factors evaluation of loaded cracked structures.
\end{abstract}

Keywords: Cracks, caustics, stress intensity factors, plastic zone.

\section{INTRODUCTION}

The optical method of caustics is suitable for the experimental study of singularities in stress fields created either by geometric discontinuities or by loading. For many crack problems, the stress intensity factors at the crack-tip were calculated by caustics [1-3]. According to the method of caustics, the light rays impinging normally at the thin plate are partly reflected from either the front or the rear faces of the plate. The reflected rays are deviated because of the important constraint of the plate at the vicinity of the applied concentrated load and the significant variation of the refractive index there. The deviated light rays, when projected on a reference screen, are concentrated along a singular curve which is strongly illuminated and forms a caustic. It was proved that the shape and size of the caustic depend on the stress singularity at the point of application of the load. The initiation of crack propagation is essential for security problems. The study of initiation of crack propagation is presupposed the extension of the plastic zone at crack tip. Among the experimental techniques used to investigate the neighborhood of a crack tip, optical methods are widely employed, as the interferometry, photoelasticity and caustics. The method of reflected and transmitted caustics has been used to determine the static and dynamic stress intensity factors for elastic materials, Manogg [1], Theocaris [2], Papadopoulos [3], Spitas et. al. [4] and Badalouka et al. [5]. Recent work, Unger et al. [6] and Hedans et al. [7], has demonstrated that the method of caustics can be used to study the plasticity around the crack tip. The aim of this work is to study the dependence of the caustic shape on the plastic zone and to show that the optical method of caustics can be used to determine the extension of the plastic zone ahead of the crack tip.

*Address correspondence to these authors at the Department of Engineering Science, Section of Mechanics, National Technical University of Athens, GR-157 73, Zografou Campus, Athens, Greece;

Tel: +30 2107721228 ; Fax: +30 2107721302 ;

E-mails: vvada9@yahoo.gr,gpad@central.ntua.gr

\section{METHOD OF REFLECTED CAUSTICS}

The optical method of caustics is able to transform the stress singularity into an optical singularity, using the geometric reflection laws. For divergent light beam the reflected light rays from front (f) and rear (r) plate faces form two caustics, the caustic (f) and the caustic (r). For a cracked isotropic elastic specimen, the parametric equations of the two caustics are [3]:

Caustic (f):

$$
X_{f}=\lambda_{m} r_{o f}\left(\cos \theta-\frac{2}{3} \cos \frac{3 \theta}{2}\right)
$$$$
Y_{f}=\lambda_{m} r_{o f}\left(\sin \theta-\frac{2}{3} \sin \frac{3 \theta}{2}\right)
$$$$
X_{r}=\lambda_{m} r_{o r}\left(\cos \theta+\frac{2}{3} \cos \frac{3 \theta}{2}\right)
$$

Caustic (r)

$$
Y_{r}=\lambda_{m} r_{o r}\left(\sin \theta+\frac{2}{3} \sin \frac{3 \theta}{2}\right)
$$

where $r_{o f}$ and $r_{o r}$ are the radii of initial curves of the caustics, respectively. These radii are given by:

$$
r_{o f}=\left(\frac{3}{2} C_{f}\right)^{2 / 5} \quad \text { with } \quad C_{f}=\frac{z_{o} d c_{f} K_{I}}{\lambda_{m} \sqrt{2 \pi}}
$$

and:

$r_{o r}=\left(\frac{3}{2} C_{r}\right)^{2 / 5} \quad$ with $\quad C_{r}=\frac{2 z_{o} d c_{r} K_{I}}{\lambda_{m} \sqrt{2 \pi}}$

and:

$$
\lambda_{m}=\frac{z_{o}+z_{i}}{z_{i}}
$$

where $z_{o}$ is the distance between specimen and reference plane, $d$ is the thickness of the specimen, $\lambda_{m}$ is the magnification ratio of the experimental set-up, $z_{i}$ is the distance between specimen and light beam focus and $K_{I}$ is the stress intensity factor for the mode-I stress state. $c_{r}$ and 

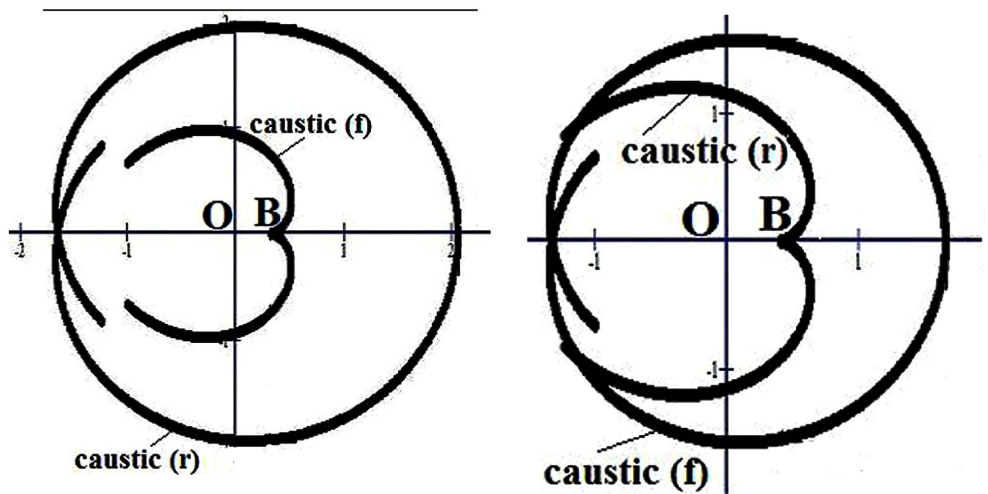

Fig. (1). Geometry of caustics (r) and (f), (a) for divergent light beam and (b) for convergent light beam.

$c_{f}$ are the material stress-optical constants. The stressoptical constant $c_{f}$ is given by:

$c_{f}=\frac{v}{E}$

where $v$ is the Poisson's ratio and $E$ is the material modulus of elasticity.

Fig. (1) illustrates the plotting of the two caustics, (r) and (f), for (a) divergent light beam and (b) convergent light beam. As it is appears from Fig. (1) the two caustics change shape depending on light beam (divergent or convergent). For the plotting, this change is corresponding to switching the (-) with $(+)$ in relations (1) and the $(+)$ with $(-)$ in relations (2).

The experimental stress intensity factor $K_{I}$ is estimated by the relation [3]:

$$
K_{I}=\frac{\sqrt{2 \pi}}{3 z_{o} d \lambda_{m}^{3 / 2} c_{r}}\left(\frac{D_{t}}{3.1702}\right)^{5 / 2}
$$

where $D_{t}$ is the maximum diameter of the caustic (r).

\section{EXPERIMENTAL PROCEDURE}

For the present investigation Lexan (PCBA) specimens with a length of $0.300 \mathrm{~m}$, a width of $0.080 \mathrm{~m}$ and a thickness of $d=0.003 \mathrm{~m}$ were used during the tensile tests. The edge crack lengths were $\alpha=0.028 m, 0.040 m, 0.056 m, 0.069 m$.

The Lexan stress optical constant was $c_{r}=2.04 \times 10^{-10} \mathrm{~m}^{2} / N$. For divergent light beam, the magnification ratio was $\lambda_{m}=4.7179$ and the distance between specimen and reference plane was $z_{0}=1.45 \mathrm{~m}$ and the distance between specimen and light beam focus was $z_{i}=0.390 \mathrm{~m}$.

\section{RESULTS AND DISCUSSION}

Fig. (2) illustrates the experimental and plotting caustics corresponding to experiments with tensile stress $\sigma=2.37 \mathrm{MPa}$ and edge crack lengths a) $\alpha=0.028 \mathrm{~m}, \mathrm{~b}$ ) $\alpha=0.040 \mathrm{~m}$, c) $\alpha=0.056 \mathrm{~m}$ and d) $\alpha=0.069 \mathrm{~m}$. As it appears in photos (c) and (d), the caustics became oval. This means that in front of the crack tip a small plastic zone was appearing. In photos (a) and (b) the ratio of the diameters of the caustics (transverse $D_{t}$ and longitudinal $D_{l}$ ) is $D_{t} / D_{l}=1.056$ as the ratio of the plotting (theoretical) caustics. The diameters ratio of the caustics in photos (c) and (d) is $D_{t} / D_{l}<1.056$. This means that the longitudinal diameter becomes bigger than the transverse diameter. The biggest longitudinal diameters is depended from the size of the created plastic zone in front of the crack tip.

Fig. (3) illustrates the experimental and plotting caustics obtained from tensile experiments for edge crack lengths $\alpha=0.040 \mathrm{~m}$ and tensile stresses a) $\sigma=4.9 \mathrm{MPa}$, b) $\sigma=6.13 \mathrm{MPa}$, c) $\sigma=8.58 \mathrm{MPa}$ and d) $\sigma=11.03 \mathrm{MPa}$.

Fig. (4) illustrates the variation of diameters ratio of the caustic versus the stresses for various edge crack lengths as obtained from tensile experiments. From these curves the effect of the size of plastic zone on the diameters of the caustics can be observed. The diameters ratio is rapidly decreasing (mainly for $\alpha=0.069 \mathrm{~m}$ ) because the longitudinal diameter of the caustic was increased by the plastic zone increased.

Fig. (5) illustrates the percentage variation of the caustic shape (deformation) ( $D \%$ ) versus the stresses for various edge crack lengths as obtained from tensile experiments. The value size of the deformation $D$ was calculated by the relation:

$D=\frac{\left(D_{t} / D_{l}\right)_{i}-\left(D_{t} / D_{l}\right)_{0}}{\left(D_{t} / D_{l}\right)_{0}}$

From these curves the effect of the crack length on the values of caustic shape deformation $D$ can be observed (mainly for $\alpha=0.069 m$ ).

Fig. (6) illustrates the percentage variation of the permanent caustic shape deformation per cent $\left(D_{\text {perm. }} \%\right)$ versus the crack length as obtained from tensile experiments and from caustics at the crack tip after unloading the specimens. The size of the permanent caustic shape deformation $D_{\text {perm. }}$. was calculated by relation (8). From this curve the effect of crack length on the size of permanent caustic shape deformation can be observed.

Fig. (7) illustrates the variation of the percentage plastic zone size $\left(R_{p l . z o n} \%\right)$ in front of the crack tip versus stresses 


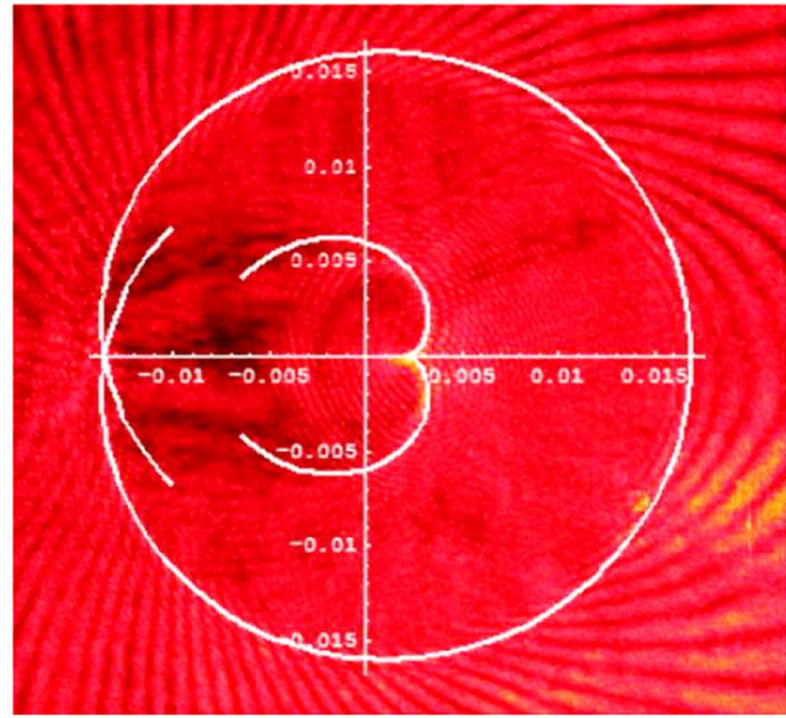

(a)

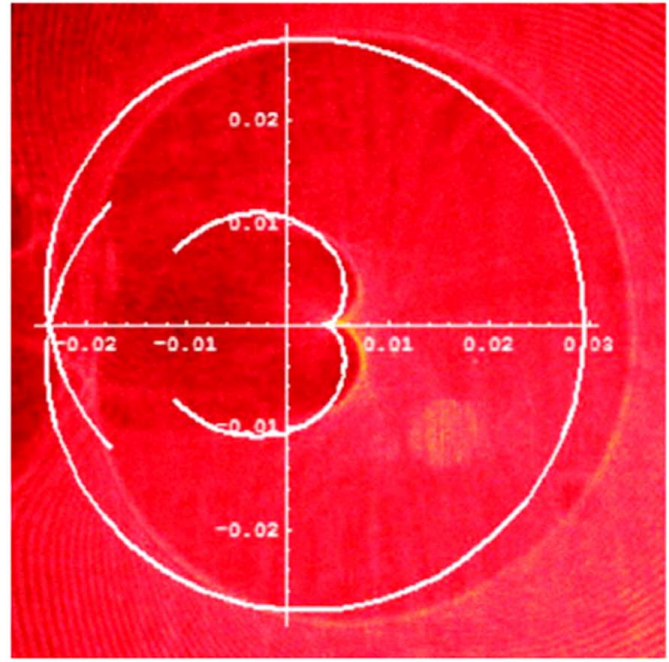

(c)

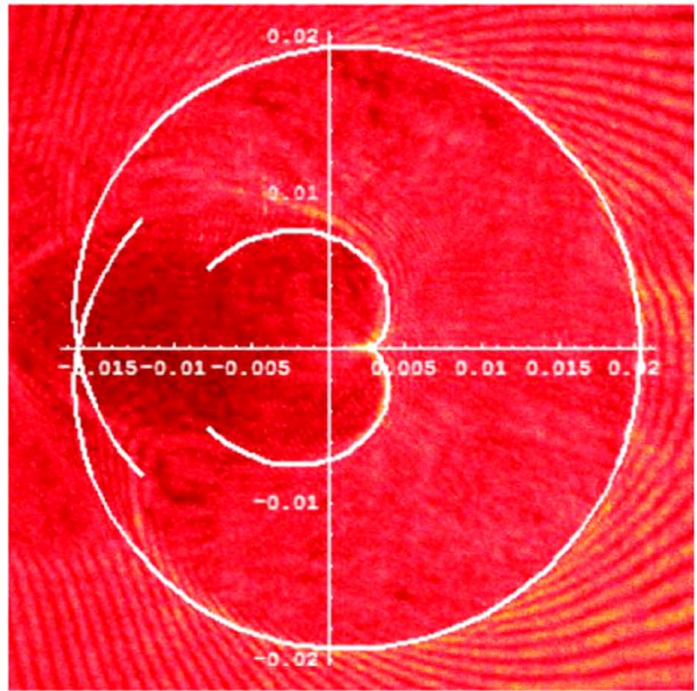

(b)

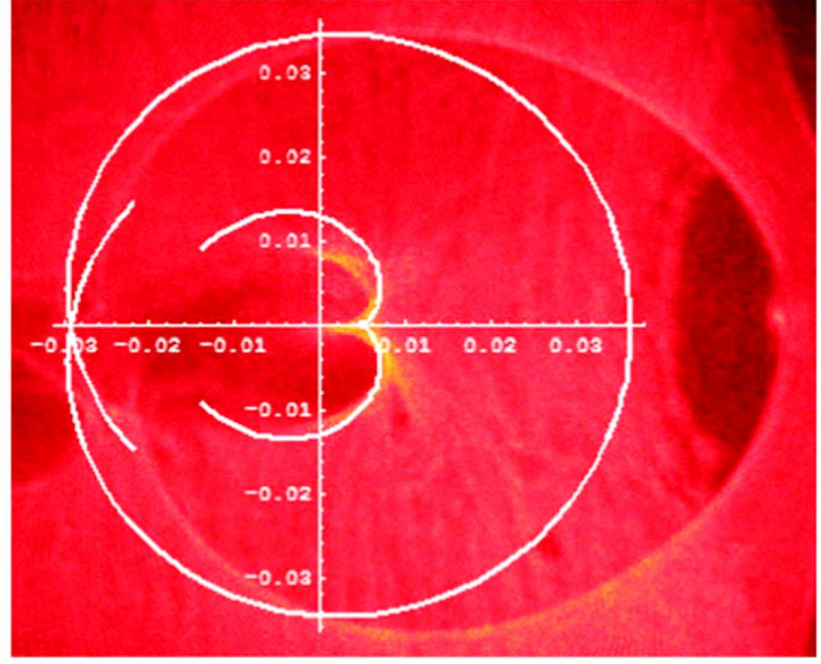

\section{(d)}

Fig. (2). Experimental and plotting caustics obtained from tensile experiments for tensile stress $\sigma=2.37 M P a$ and edge crack lengths a) $\alpha=0.028 \mathrm{~m}$ b) $\alpha=0.040 \mathrm{~m}$ c) $\alpha=0.056 \mathrm{~m}$ and d) $\alpha=0.069 \mathrm{~m}$.

for various edge crack lengths as obtained from tensile experiments. The size of the plastic zone $R_{\text {pl.zon. }}$ was calculated by the relation:

$$
R_{p l . z o n .}=\frac{\Delta D_{l}}{D_{l 0}} \%
$$

From these curves the effect of stress on plastic zone size $R_{p l . z o n}$. can be observed.
Fig. (8) illustrates the variation of the experimental stress intensity factor $K_{I}^{\text {exp. }}$ versus the edge crack length for a stress $\sigma=2.37 \mathrm{MPa}$ and a specimen width $w=0.080 \mathrm{~m}$. The stress intensity factor $K_{I}^{\text {exp. }}$ was calculated by the relation (7) for crack length $\alpha=0.040 \mathrm{~m}$ and specimen width $w=0.080 \mathrm{~m}$. From this curve a nearly linear variation can be observed for great crack lengths. While, for small crack length a non-linear variation can be observed because a triaxiality stress state was created. 


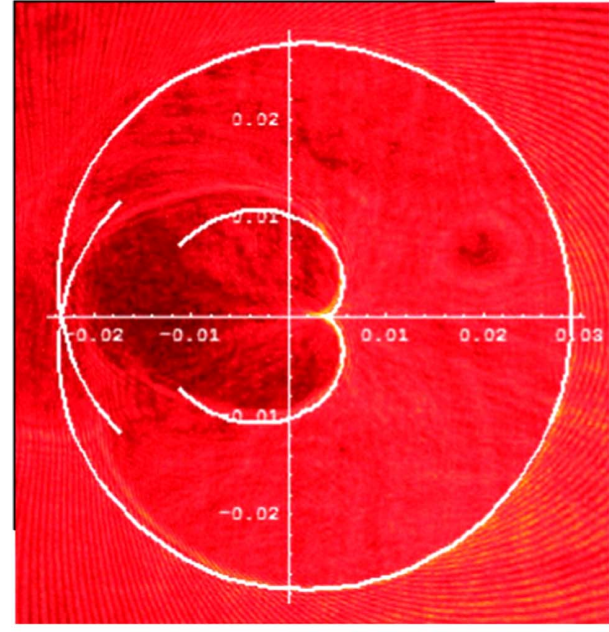

(a)

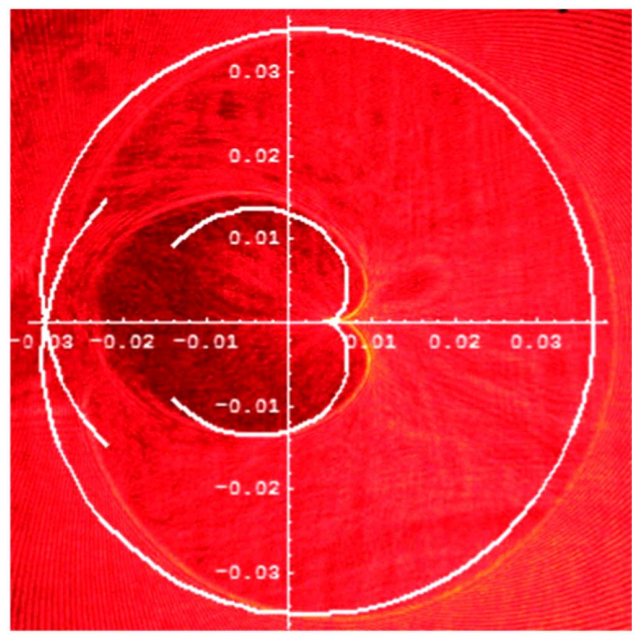

(c)

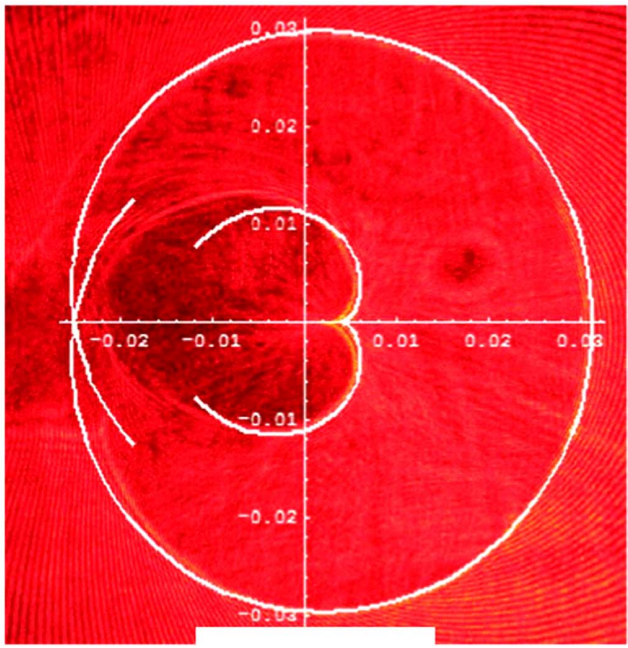

(b)

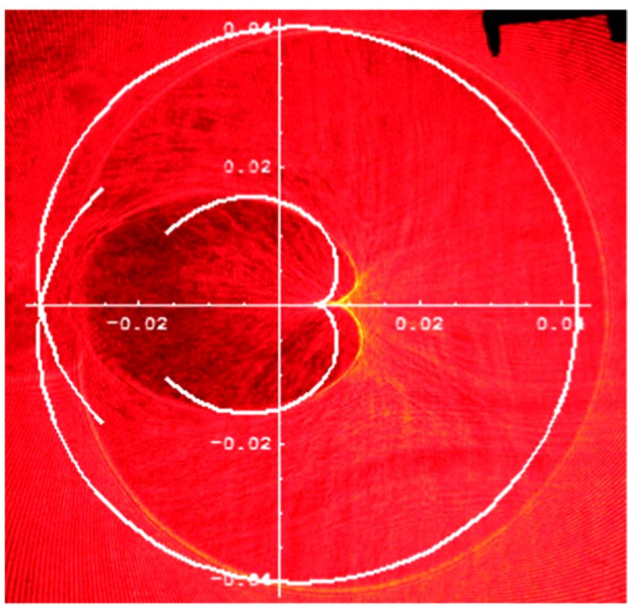

(d)

Fig. (3). Experimental and plotting caustics obtained from tensile experiments for edge crack lengths $\alpha=0.040 \mathrm{~m}$ and tensile stresses a) $\sigma=4.9 \mathrm{MPa}$ b) $\sigma=6.13 \mathrm{MPa}$ c) $\sigma=8.58 \mathrm{MPa}$ and d) $\sigma=11.03 \mathrm{MPa}$.

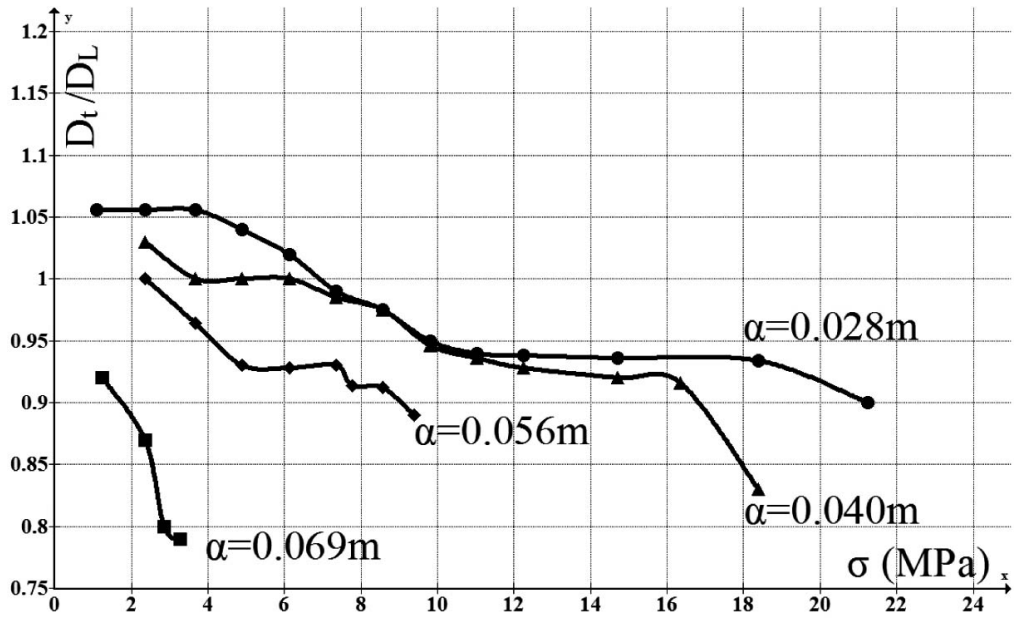

Fig. (4). Variation of the caustic diameter ratio versus stresses. 


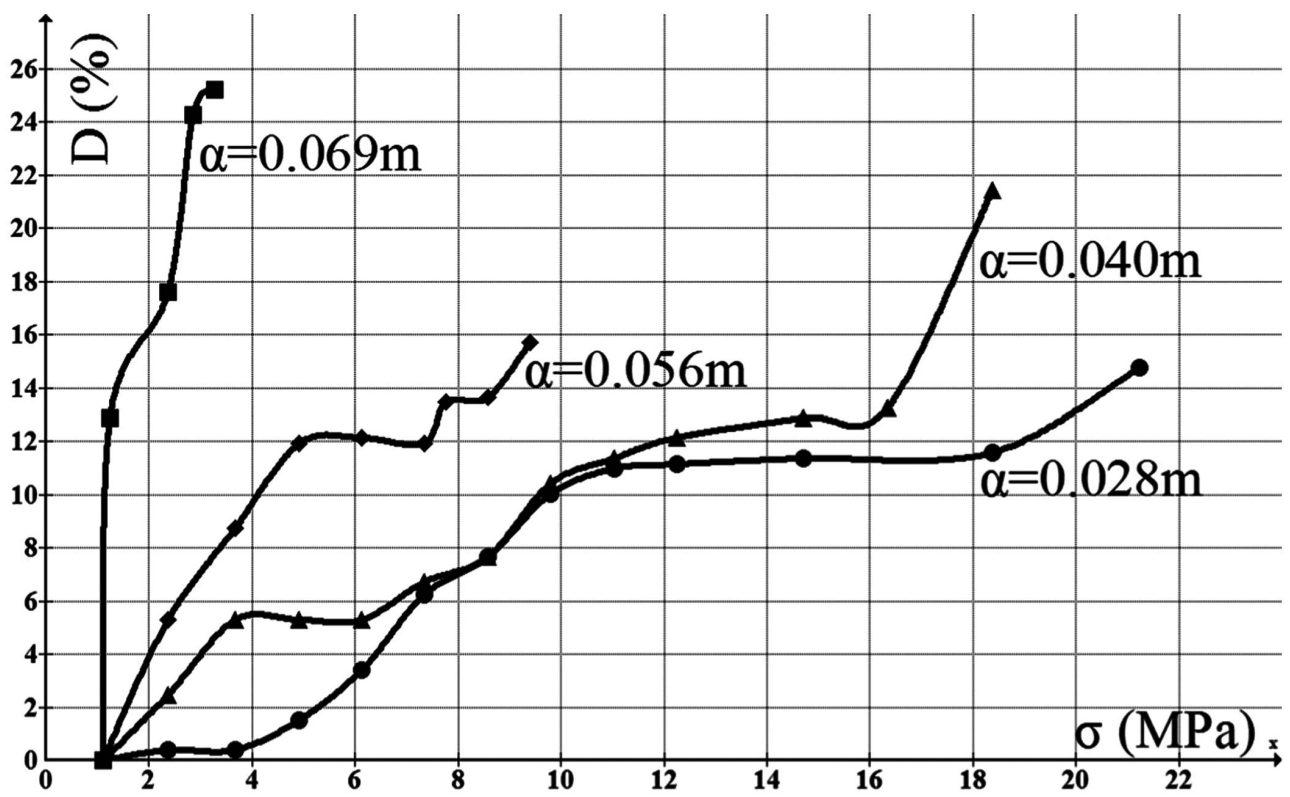

Fig. (5). Variation of the caustic shape deformation per cent $(D \%)$ versus stresses for various edge crack lengths.

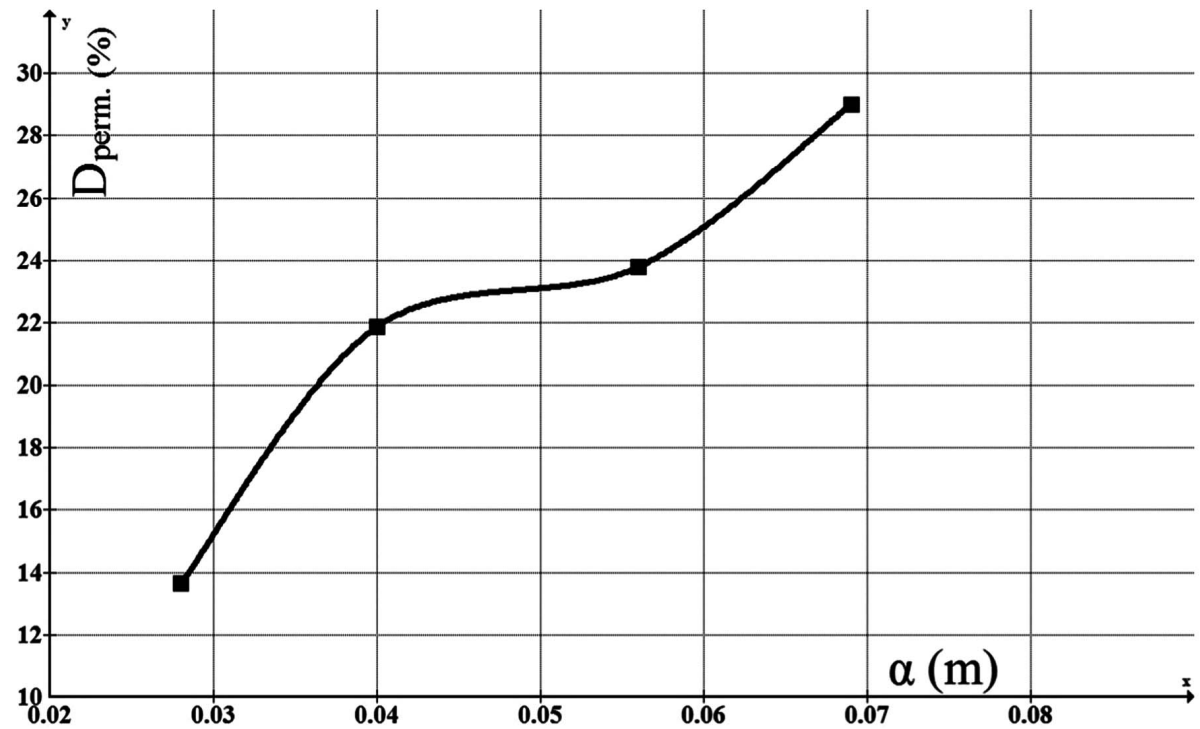

Fig. (6). Variation of the permanent caustic shape deformation per cent ( $D_{\text {perm. }} \%$ ) versus crack length.

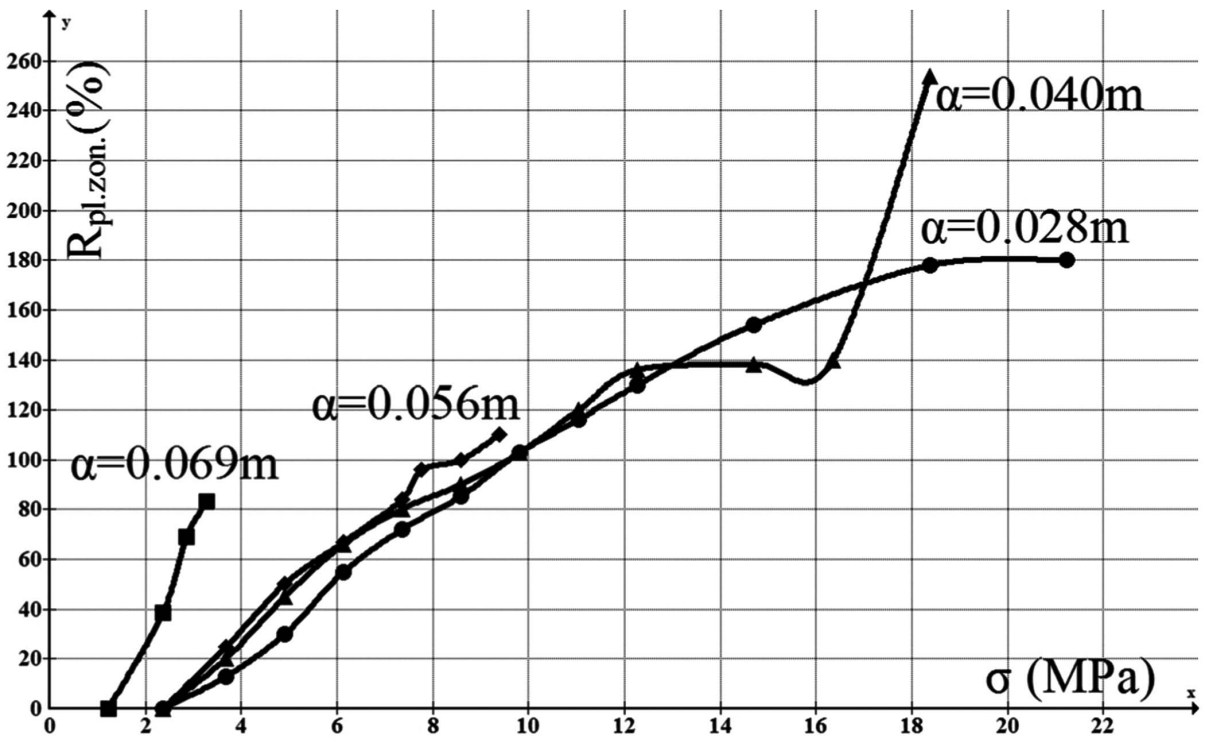

Fig. (7). Variation of the plastic zone size per cent $\left(R_{p l . z o n .} \%\right)$ in front of the crack tip versus stresses for various edge crack lengths. 


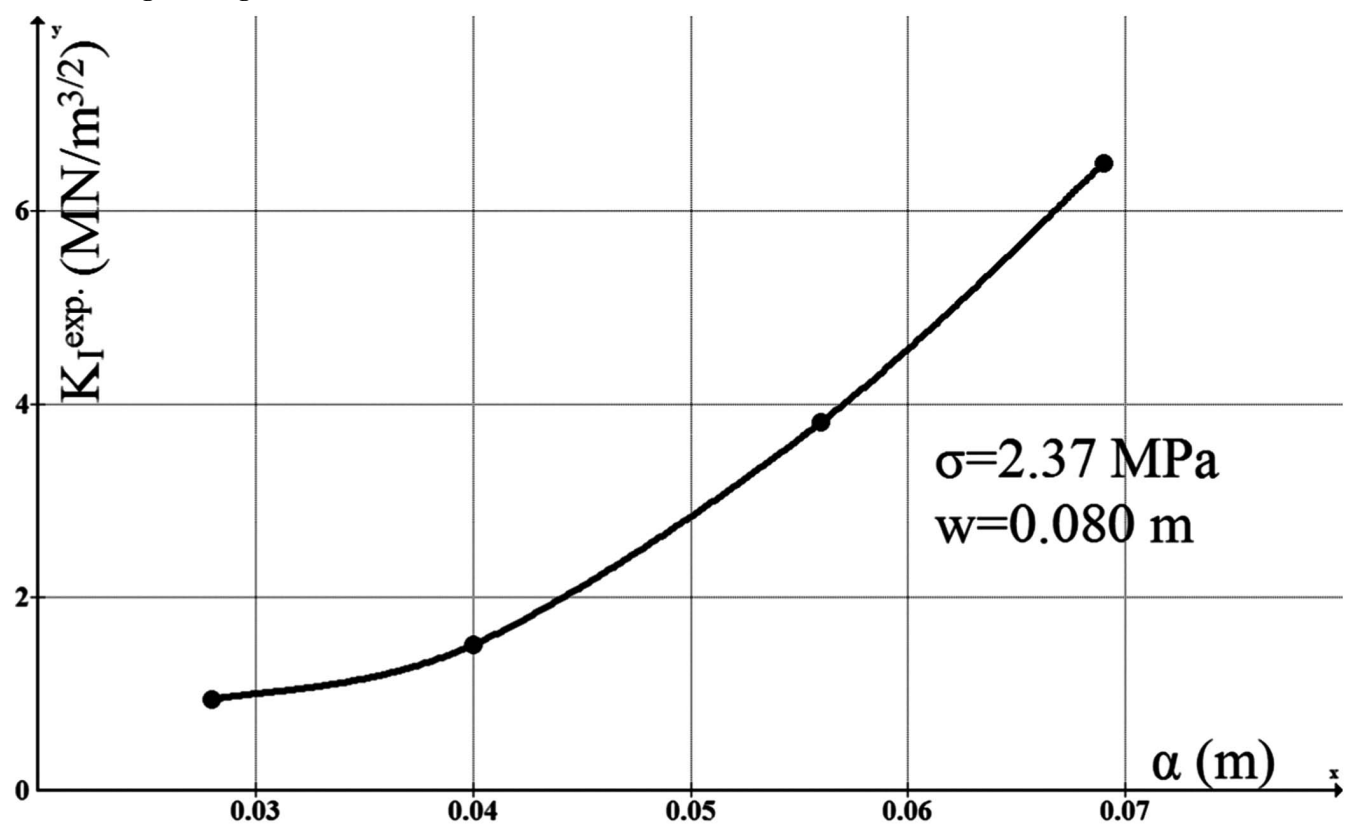

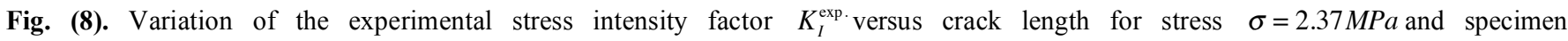
width $w=0.080 m$.

Fig. (9) illustrates the variation of the experimental stress

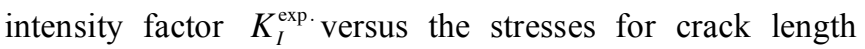
$\alpha=0.040 \mathrm{~m}$ and specimen width $w=0.080 \mathrm{~m}$. The stress intensity factor $K_{I}^{\text {exp. }}$ was calculated by the relation (7). From this curve a nearly linear variation can be observed for high stresses.

Fig. (10) illustrates the variation of the experimental stress intensity correction factor $f$ versus the ratio of crack length and specimen width $(\alpha / w)$ for a stress $\sigma=2.37 \mathrm{MPa}$.
The stress intensity correction factor $f$ was calculated by the relation:

$$
f=\frac{K_{I}^{\text {exp }}}{\sigma \sqrt{\pi \alpha}}
$$

From this curve a nearly linear variation can be observed for high ratios $(\alpha / w)$. While, for small ratios a non-linear variation can be observed because a triaxiality stress state was created.

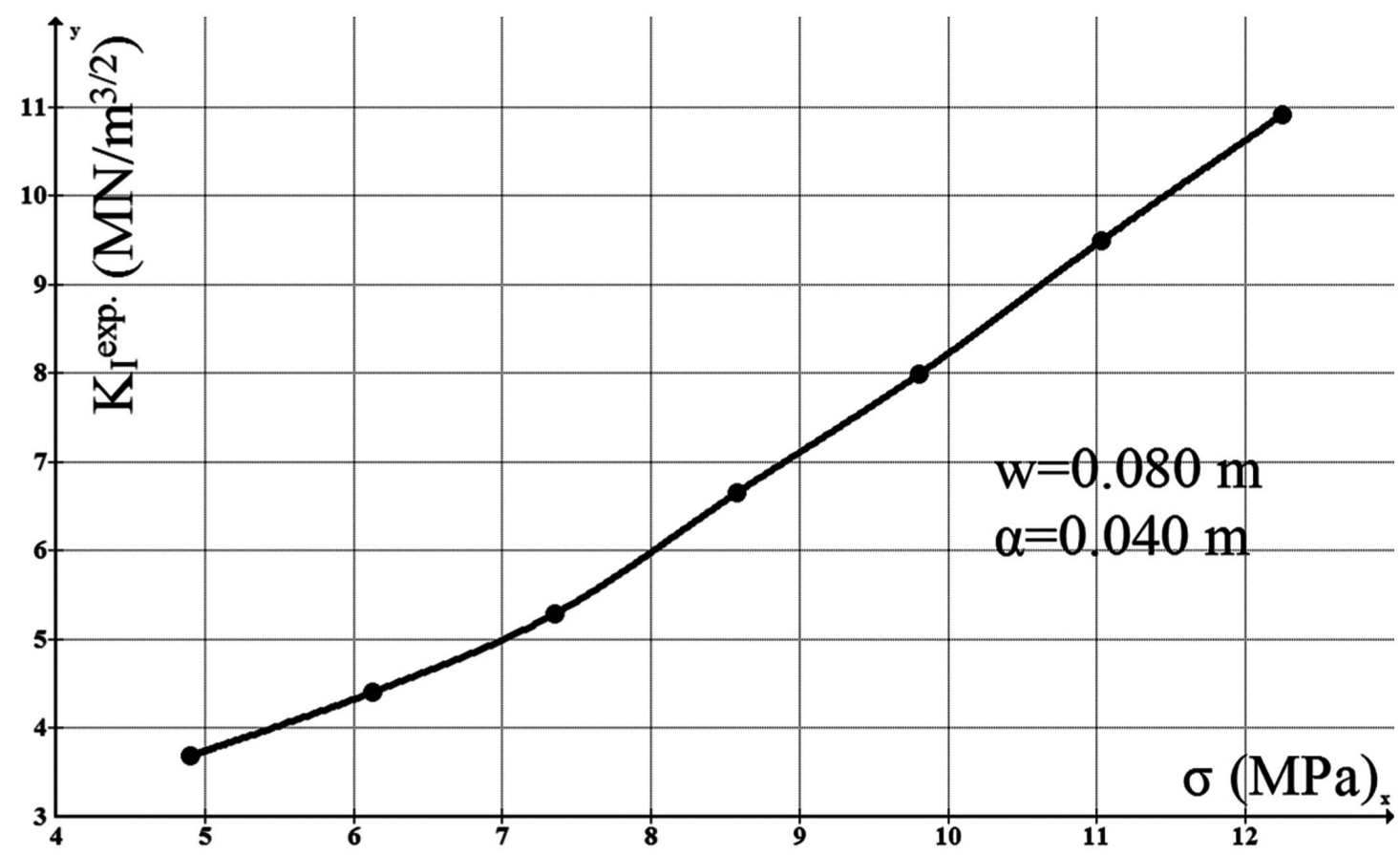

Fig. (9). Variation of the experimental stress intensity factor $K_{I}^{\text {exp. }}$ versus the stresses for crack length $\alpha=0.040 \mathrm{~m}$ and specimen width $w=0.080 m$. 


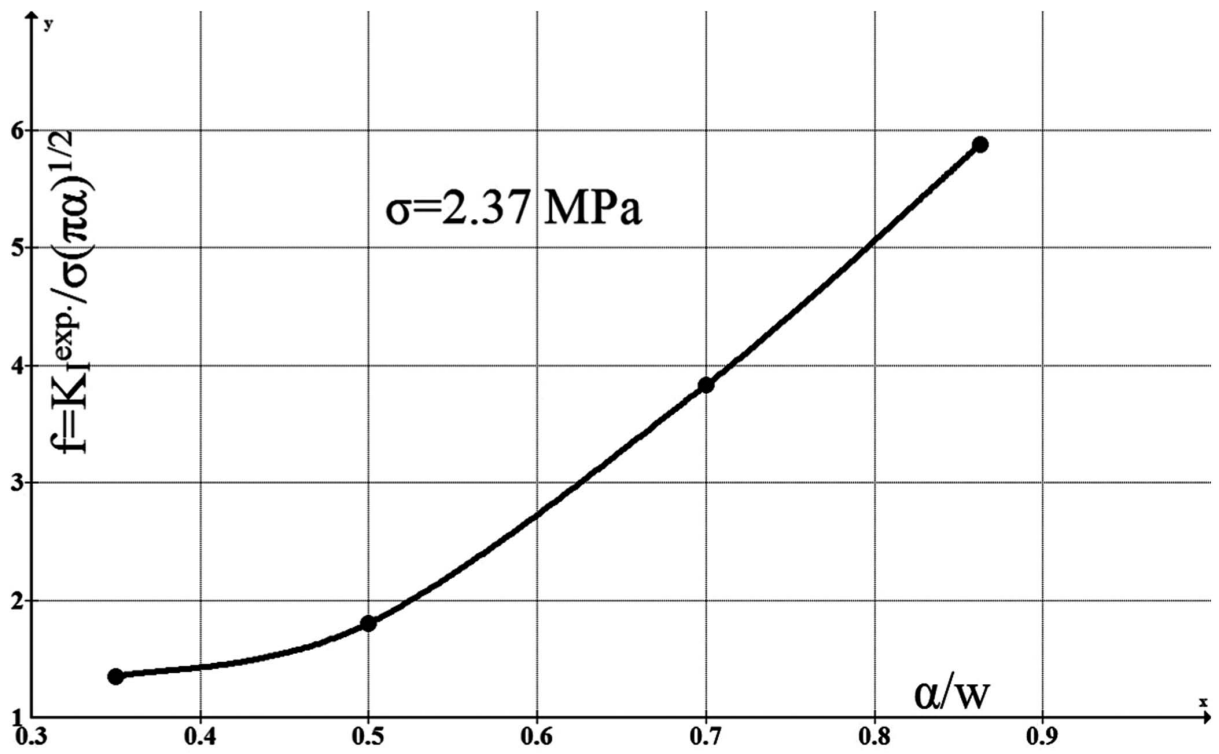

Fig. (10). Variation of the experimental stress intensity correction factor $f$ versus the ratio of crack length and specimen width for stress $\sigma=2.37 M P a$.

\section{CONCLUSIONS}

The aim of this work is to study the dependence of the caustic shape on the plastic zone and to show that the optical method of caustics can be used to determine the extension of the plastic zone ahead of the crack tip, on a ductile plate loaded in mode I. From this experimental study it is possible to conclude that the shape of caustics was deformed by crack length, stresses and plastic zone at crack tip. The plastic zone size depends on caustics diameters and mainly on the longitudinal diameter. The plastic zone variation was calculated by the variation of the caustic longitudinal diameter. This plastic zone variation depended on the state of stresses, the crack length and the materials. The stress intensity factor was calculated from the transverse diameter of the caustic. The stress intensity variation was linear for high values of stresses and crack lengths, while it was nonlinear for small stresses and crack lengths because of the triaxiality at crack tip. The experimental stress intensity correction factor was calculated for various crack lengths. This experimental study shows that the caustic optical method can be used to give a non destructive calculation of the plasticity state of a loaded cracked ductile plate.

\section{ACKNOWLEDGEMENT}

Declared none.

\section{CONFLICT OF INTEREST}

Declared none.

\section{REFERENCES}

[1] P. Manogg, Anwendung der Schattenoptik zur Untersuchung des Zerreissvorgangs von Platten. Dissertation 4/64, Universitaet Freiburg, 1964.

[2] P.S. Theocaris, "Local yielding around a crack-tip in plexiglas", $J$. Appl. Mech. ASME, vol. 37, pp. 409-415, 1970.

[3] G.A. Papadopoulos, "The Experimental Method of Caustics and the Det.-Criterion of Fracture", G.A. Papadopoulos ed., Fracture Mechanics, Springer-Verlag, London, 1993.

[4] V. Spitas, G.A. Papadopoulos, C. Spitas, and T. Costopoulos, "Experimental investigation of load sharing in multiple gear tooth contact using the stress-optical method of caustics". Strain, vol. 47, pp. e227-e233, 2011.

[5] B.G. Badalouka and G.A. Papadopoulos, "Range of applicability of the method of caustics around holes". Strain, vol. 42, pp. 283290, 2006.

[6] D.J. Unger, "Perfectly plastic caustics for the opening mode of fracture". Theor. Appl. Fract. Mech., vol. 44, pp. 82-94, 2005.

[7] S. Hedans, O. Pop, V. Valle and M. Cottron, "FE and experimental investigation with shadow optical method for measuring plastic zone in a ductile cracked plate. Strain, vol. 47, pp. e142-e147, 2011. 